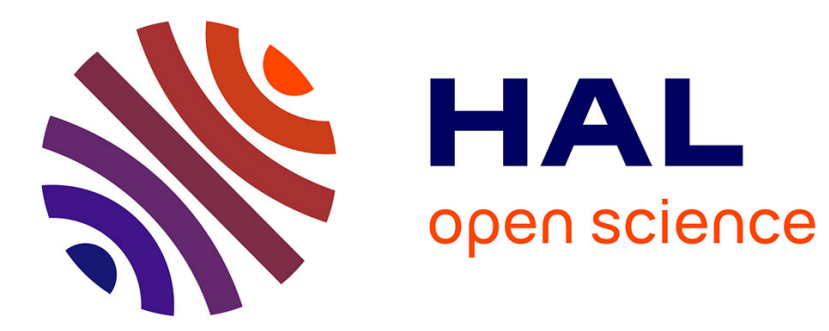

\title{
Stability analysis of a regulated oxygen mask
}

Geoffray Battiston, Dominique Beauvois, Gilles Duc, Emmanuel Godoy

\section{To cite this version:}

Geoffray Battiston, Dominique Beauvois, Gilles Duc, Emmanuel Godoy. Stability analysis of a regulated oxygen mask. ICINCO2018, Jul 2018, Porto, Portugal. 10.5220/0006848203170324 . hal02963601

\section{HAL Id: hal-02963601 \\ https://hal.science/hal-02963601}

Submitted on 10 Oct 2020

HAL is a multi-disciplinary open access archive for the deposit and dissemination of scientific research documents, whether they are published or not. The documents may come from teaching and research institutions in France or abroad, or from public or private research centers.
L'archive ouverte pluridisciplinaire HAL, est destinée au dépôt et à la diffusion de documents scientifiques de niveau recherche, publiés ou non, émanant des établissements d'enseignement et de recherche français ou étrangers, des laboratoires publics ou privés. 


\title{
Stability analysis of a regulated oxygen mask
}

\author{
Geoffray Battiston, Dominique Beauvois, Gilles Duc and Emmanuel Godoy \\ Laboratoire des Signaux et Systèmes (L2S), Centrale-Supélec, CNRS, Université Paris-Sud, Université Paris-Saclay, 3 rue \\ Joliot Curie, 91192 Gif sur Yvette, cedex, France \\ Geoffray.Battiston@centralesupelec.fr,Dominique.Beauvois@centralesupelec.fr, Gilles.Duc@centralesupelec.fr, \\ Emmanuel.Godoy@centralesupelec.fr
}

\begin{abstract}
Keywords: $\quad$ Stability Analysis, Pneumatic systems.
Abstract: We analyse the stability of a regulated oxygen mask distributing oxygen in response to an inhalation demand. The mask pressure exhibits troublesome vibrations after the demand reaches a certain flow value. Starting from a simple nonlinear model, we perform a local linear stability analysis which highlights that the real part of two eigenvalues is positive when this system is chattering. We propose then adjustments of some parameters of this purely mechanical system in order to avoid this phenomenon. These adjustments have been tested and validated experimentally.
\end{abstract}

\section{INTRODUCTION}

In an airplane, in order to avoid hypoxia accidents which can be due to fire or cabin depressurization, some international aviation agencies (like NATO, 1991 or EASA, 2012) impose the availability and performance of an oxygen supply for aircraft pilots. We analyze the stability of a regulated oxygen mask distributing oxygen in response to an inhalation demand. The mask pressure exhibits troublesome vibrations after the demand reaches a certain flow value. Starting from a simple nonlinear model, we perform a local linear stability analysis which highlights that the real part of two eigenvalues is positive when this system is chattering. We propose then adjustments of some parameters of this purely mechanical system in order to avoid this phenomenon.

The regulated oxygen mask studied in this paper satisfies all the requirements but still presents a mask pressure vibration issue which can disturb an inhaling pilot. For comfort reasons, these vibrations must be suppressed. The regulator of the mask is made of purely mechanical elements and for cost reasons we can only adjust its physical parameters.

As in many studies of pneumatic systems, we cope with relief valves which introduce amplitude constraints in addition to nonlinearities linked to the nature of the flows in the pneumatic circuit. It is known that valves coupled with a phase shifting element like a long tube or volume can be affected with various dynamical behaviours like chattering, fluttering, pressure surge, etc. (a complete review for pressure relief valves can be found in Hös et al., 2017). Chattering, which concerns us the most here, is basically the situation where the valve starts touching its support. The study of the chattering phenomenon is linked to this grazing bifurcation and can be found in multiple papers (see for instance, Budd, 1996, Chin et al., 1994, Casas et al., 1996 or Molenaar et al., 2001) or books (see Bernardo et al., 2008).

The classical method for such a study is, usually first, to perform a stability analysis of a linearized model of this nonlinear system to find the possible instabilities, Hopf bifurcations (see for instance, Maeda, 1970, MacLeod, 1985), secondly to eventually use nonlinear tools (normal form reduction, center manifold reduction, Lyapunov exponents, etc.) to analyze the trajectory of the system variables until a grazing bifurcation appears (Hayashi et al., 1997, Licsko et al., 2009). Thirdly, the grazing bifurcation can be studied by finding a Poincaré map which describes the essential dynamics of the oscillating valve that collides with its support (see the suggestions about chattering in the papers cited above). In this paper, we will only focus on the first step, which is finding the conditions provoking the chattering effect, and see if it is possible to find any stability condition for the regulated mask and 
how it can be fulfilled by modifying some physical parameters.

Note that a first idea is to perform the linear stability analysis on a very accurate model to understand accurately the sensitivity of the vibrations and determine conditions on the parameters for their apparition. However it appeared we can still get a similar analysis and accurate conclusions with a simplified model which we present here.

The model equations are presented in section 2 . A stability analysis for this model is performed in section 3 in order to find the conditions of stability and which parameters must be modified to satisfy them. In section 4 , the results of one modification on the regulator are shown and the direct consequence for the pilot is studied in section 5 .

\section{MODEL OF THE OXYGEN REGULATOR}

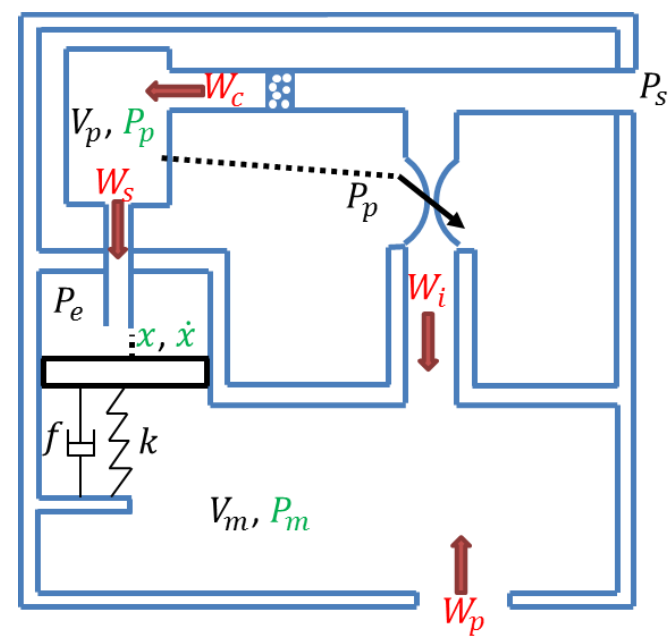

Figure 1: Definition sketch of the mathematical model. In red, the mass flows, in green, the future state variables. The dashed line represents a pressure measurement.

A schematic diagram of the oxygen mask is given on Fig.1: the flow demand $W_{p}$ is detected with a comparator balancing a constant rest pressure $P_{e}$ and the mask pressure $P_{m}$ evaluated in the mask volume $V_{m}$ where the pilot breathes; its behaviour is described by the virtue of a mass conservation equation, which simply integrates all the mass flow values entering the corresponding volume:

$$
\dot{P_{m}}=\frac{r T}{V_{m}}\left(W_{i}+W_{p}\right)
$$

where the mass flow $W_{p}$ of the pilot's inhalation is negative. $W_{i}$ is described later. $r$ represents the oxygen gas constant and $T$ the gas temperature (whose variation in the whole pneumatic circuit is neglected for simplicity).

The comparator is basically modelled as a spring mass system with viscous friction (damping $f$ ) and positive preconstraint $x_{0}$, consisting in a spring of stiffness $k$ and a rigid disk of mass $m$, on which three static pressures applies, $P_{e}, P_{m}(t)$ and $P_{p}(t)$ over two distinct surfaces $S_{m}$ and $S_{s}$. For a displacement $x$ greater than 0 , we obtain:

$$
\begin{gathered}
m \ddot{x}=-f \dot{x}-k\left(x+x_{0}\right)+S_{m}\left(P_{e}-P_{m}\right) \\
+S_{s}\left(P_{p}-P_{m}\right)
\end{gathered}
$$

Before an inhalation, the comparator is closed, and $P_{p}=P_{s}$ (it will become obvious later with equations (6) and (7)). Because of the spring preconstraint, it opens when the difference $P_{e}-$ $P_{m}$ equals some threshold $d P_{0}$ which corresponds to the limit of opening $x=0$ and is given by:

$$
S_{m} d P_{0}+S_{s}\left(P_{s}-P_{e}+d P_{0}\right)-k x_{0}=0
$$

We subtract the last two equations and neglect $P_{e}-d P_{0}-P_{m}$ in front of $P_{p}-P_{s}$ (which is justified experimentally) and get the equation for the moving comparator when $x>0$ :

$$
\begin{aligned}
m \ddot{x}=-f \dot{x}-k x & +S_{m}\left(P_{e}-P_{m}-d P_{0}\right) \\
& +S_{s}\left(P_{p}-P_{s}\right)
\end{aligned}
$$

At $x=0$, the comparator enters in contact with a tube which may lead to a bounce (the chattering effect) or a simple closing. This acts as a lower limit for a saturation effect but also as a threshold to open the comparator.

The vertical displacement $x$ of the comparator can open this evacuation tube (in which the propagative effects are neglected due to its small length), in which the pressure will be approximated by $P_{p}$ (due to the small speed of the oxygen flow inside it).

For physicists, this system can be qualified as an extended flapper nozzle valve due to the presence of the $S_{m}$ term. For reasons linked to the Reynolds number of the flow going through this dispositive, its modeling is in general far trickier that the simple proportional term $S_{s}\left(P_{p}-P_{m}\right)$ as above (see McCloy and Martin, 1980). In this case, all the nonlinearities linked to this kind of systems (Reynolds number, flat of the support) will be caught into $S_{S}$ which in the case 
of our study will be considered constant and estimated via experimental measurements.

The tube is connected to a small volume, the whole gathered into a mathematical volume $V_{p}$. The mass flows $W_{s}$ leaves $V_{p}$ thanks to the opening of the comparator, and the flow $W_{c}$ enters $V_{p}$ to refill it.

$W_{s}$ is determined with a Saint-Venant law for sonic flows:

$$
W_{s}=2 \pi R_{s} x P_{p} \sqrt{\frac{\gamma}{r T}\left(\frac{2}{\gamma+1}\right)^{\frac{\gamma+1}{\gamma-1}}}=k_{s} x P_{p}
$$

with $R_{s}$ the support internal radius and $\gamma$ the Laplace coefficient.

$W_{c}$ is more accurately modelled with an isothermal Hagen-Poiseuille law in which the pressure $P_{S}$ is the constant regulator supply pressure:

$$
W_{c}=k_{c}\left(P_{s}^{2}-P_{p}^{2}\right)
$$

Hence the mass conservation equation in volume $V_{p}$ can be written as:

$$
\dot{P_{p}}=\frac{r T}{V_{p}}\left(W_{c}-W_{s}\right)
$$

In this configuration, physics always imposes the working region: $P_{s} \geq P_{p}$, that is $W_{c} \geq 0$. Indeed, if $x=0$, then $W_{s}=0$ and $P_{p}$ increases until it reaches $P_{s}$. Moreover, the regulator never starts with a pressure $P_{p}$ greater than $P_{S}$.

Now, we introduce the regulating actuator, which delivers the mass flow $W_{i}$ filling the mask volume depending on the difference between $P_{s}$ and $P_{p}$ :

$$
W_{i}=\left\{\begin{aligned}
k_{i}\left(P_{s}-P_{p}-P_{t}\right), & P_{p} \leq P_{s}-P_{t} \\
0, & P_{p}>P_{s}-P_{t}
\end{aligned}\right.
$$

where $P_{t}$ is the threshold for $P_{s}-P_{p}$ to start delivering a mass flow.

There are finally one threshold for the comparator and one for the actuator. Indeed, these thresholds have a destabilizing effect due to the mechanical delay imposed to the system response. From experimental observations, the instability we seek to prevent only occurs when these two thresholds are overcome. Nevertheless, if we wanted to characterize the chattering phenomenon, we would have to take them into account.

\section{STABILITY ANALYSIS}

We write the state vector $X$ of this system, the input vector $U$, and the external conditions vector $E$ as:

$$
X=\left[\begin{array}{c}
x \\
\dot{x} \\
P_{m} \\
P_{p}
\end{array}\right], \quad U=W_{p}, \quad E=\left[\begin{array}{c}
P_{e} \\
P_{s} \\
P_{t} \\
d P_{0}
\end{array}\right]
$$

From Eq. (1) to (8), the state equations are deduced:

$$
\dot{X}=\left[\begin{array}{c}
\frac{\dot{x}}{m} x-\frac{f}{m} \dot{x}-\frac{S_{m}}{m} P_{m}+\frac{S_{s}}{m} P_{p}+H \\
-k_{i} \frac{r T}{V_{m}} P_{p}+\frac{r T}{V_{m}} W_{p}+\frac{r T}{V_{m}} k_{i}\left(P_{s}-P_{t}\right) \\
\frac{r T}{V_{p}}\left(-k_{c} P_{p}^{2}-k_{s} x P_{p}\right)+\frac{r T}{V_{p}} k_{c} P_{s}^{2}
\end{array}\right]
$$

with $H=S_{m}\left(P_{e}-d P_{0}\right) / m-S_{S} P_{s} / m$. Writing state variables at the equilibrium with a bar, the equilibrium conditions for this system are:

$$
\begin{gathered}
k \bar{x}=S_{m}\left(P_{e}-\overline{P_{m}}-d P_{0}\right)+S_{s}\left(\overline{P_{p}}-P_{s}\right) \\
k_{i}\left(P_{s}-\overline{P_{p}}-P_{t}\right)=-W_{p}=\overline{W_{i}} \\
k_{c}\left(P_{s}^{2}-{\overline{P_{p}}}^{2}\right)=k_{s} \bar{x} \overline{P_{p}}
\end{gathered}
$$

That is:

$$
\begin{gathered}
\overline{P_{m}}=\left(S_{m}\left(P_{e}-d P_{0}\right)+S_{s}\left(\overline{P_{p}}-P_{s}\right)\right. \\
-k \bar{x}) / S_{m} \\
\overline{P_{p}}=-\overline{W_{i}} / k_{i}+P_{s}-P_{t} \\
\bar{x}=k_{c} / k_{s}\left(P_{s}^{2} / \overline{P_{p}}-\overline{P_{p}}\right)
\end{gathered}
$$

We linearize equations (10) around these equilibrium points and get the Jacobian $J$ of this system:

$$
\begin{gathered}
J= \\
{\left[\begin{array}{cccc}
0 & 1 & 0 & 0 \\
-\frac{k}{m} & -\frac{f}{m} & -\frac{S_{m}}{m} & \frac{S_{s}}{m} \\
0 & 0 & 0 & -\frac{r T}{V_{m}} k_{i} \\
-\frac{r T}{V_{p}} k_{s} P_{p} & 0 & 0 & -\frac{r T}{V_{p}}\left(2 k_{c} P_{p}+k_{s} x\right)
\end{array}\right]}
\end{gathered}
$$

The characteristic equation of the linearized system is: 


$$
\chi(\lambda)=\lambda^{4}+a_{3} \lambda^{3}+a_{2} \lambda^{2}+a_{1} \lambda+a_{0}
$$

with:

$$
\begin{gathered}
a_{3}=-J(2,2)-J(4,4) \\
a_{2}=J(2,2) J(4,4)-J(2,1) \\
a_{1}=J(2,1) J(4,4)-J(4,1) J(2,4) \\
a_{0}=-J(4,1) J(2,3) J(3,4)
\end{gathered}
$$

Those four coefficients are positive. It can be noticed using (16) that:

$$
\begin{aligned}
J(4,4)=-\frac{r T}{V_{p}}\left(2 k_{c} \overline{P_{p}}+k_{s} \bar{x}\right) \\
=-\frac{r T k_{c}}{V_{p}}\left(\overline{P_{p}}+\frac{P_{s}{ }^{2}}{\overline{P_{p}}}\right)
\end{aligned}
$$

the derivative of which is:

$$
\frac{\partial J(4,4)}{\partial P_{p}}=-\frac{r T k_{c}}{V_{p}}\left(1-\frac{P_{s}^{2}}{{\overline{P_{p}}}^{2}}\right)
$$

(15) shows that the steady state value $\overline{P_{p}}$ decreases with $\overline{W_{i}}=-\mathrm{W}_{p}$ below $P_{s}$ i.e. the derivative (24) is strictly positive when the regulator is delivering oxygen. So $J(4,4)$ is then strictly increasing with the demand once $P_{p}<P_{s}$.

The Routh criterion is now used to infer the conditions for stability of the linearized model. The five Routh terms of the first column of the Routh chart are:

$$
\begin{gathered}
1 \\
a_{3} \\
a_{2}-\frac{a_{1}}{a_{3}} \\
a_{1}\left(\frac{a_{0} a_{3}}{a_{1}}+\frac{a_{1}}{a_{3}}-a_{2}\right) /\left(\frac{a_{1}}{a_{3}}-a_{2}\right) \\
a_{0}
\end{gathered}
$$

Routh terms 1, 2 and 5 are positive, so that stability is guaranteed if terms 3 and 4 are positive too. Routh term 4 is written under the given form to make appear Routh term 3.

A detailed calculation gives:

$$
\begin{aligned}
a_{2}-\frac{a_{1}}{a_{3}} & \frac{k f}{m^{2}}+\frac{f^{2}}{m^{2}} \frac{r T k_{c}}{V_{p}}\left(\overline{P_{p}}+\frac{P_{s}^{2}}{\overline{P_{p}}}\right) \\
= & \frac{f}{m}+\frac{r T}{V_{p}} k_{c}\left(\overline{P_{p}}+\frac{P_{s}^{2}}{\overline{P_{p}}}\right) \\
+ & \frac{-\frac{r T}{V_{p}} k_{s} \frac{S_{s}}{m} \overline{P_{p}}+\frac{f}{m}\left(\frac{r T}{V_{p}} k_{c}\left(\overline{P_{p}}+\frac{P_{s}^{2}}{\overline{P_{p}}}\right)\right)^{2}}{\frac{f}{m}+\frac{r T k_{c}}{V_{p}}\left(\overline{P_{p}}+\frac{P_{s}^{2}}{\overline{P_{p}}}\right)}
\end{aligned}
$$

A change of sign can come from the third term of the numerator of (25). We choose a sufficient condition for positivity which at the same time avoids dealing with lengthy equations:

$$
\frac{k f}{m^{2}}-\frac{r T}{V_{p}} k_{s} \frac{S_{s}}{m} \overline{P_{p}}>0
$$

(26) shows that Routh term 3 is always positive if $k$ and $f$ are increased or $k_{s}, S_{s}, 1 / V_{p}, 1 / m$ are decreased.

Using the positivity of Routh term 3 and $a_{1}$, we immediately get a condition for positivity of remaining Routh term 4:

$$
\frac{a_{0} a_{3}}{a_{1}}+\frac{a_{1}}{a_{3}}-a_{2}<0
$$

Unless the first term of (27) is null, which is physically impossible since it would prevent the regulator from working, if multiplied by $a_{1} a_{3}$ condition (27) can be written as:

$$
L<R
$$

where:

$$
\begin{gathered}
L=\frac{r T k_{i}\left(S_{m}+S_{S}\right)}{V_{m} m^{2}}\left(\frac{f}{m}+\frac{r T}{V_{p}} k_{c}\left(\overline{P_{p}}+\frac{P_{s}{ }^{2}}{\overline{P_{p}}}\right)\right)^{2} \\
R=-\left(\frac{k}{m} \frac{r T}{V_{p}} k_{c}\left(\overline{P_{p}}+\frac{P_{s}{ }^{2}}{\overline{P_{p}}}\right)+\frac{r T}{V_{p}} \frac{k_{s}}{m} S_{p} \overline{P_{p}}\right)^{2} \\
+\left(\frac{k}{m} \frac{r T}{V_{p}} k_{c}\left(\overline{P_{p}}+\frac{P_{s}{ }^{2}}{\overline{P_{p}}}\right)+\frac{r T}{V_{p}} \frac{k_{s}}{m} S_{p} \overline{P_{p}}\right) \times \\
\left(\frac{f}{m} \frac{r T}{V_{p}} k_{c}\left(\overline{P_{p}}+\frac{P_{s}{ }^{2}}{\overline{P_{p}}}\right)+\frac{k}{m}\right) \times \\
\left(\frac{f}{m}+\frac{r T}{V_{p}} k_{c}\left(\overline{P_{p}}+\frac{P_{s}}{\overline{P_{p}}}\right)\right)
\end{gathered}
$$

This condition must be respected for all $\overline{P_{p}}$ and can be simplified even more using the monotonicity 
of $\overline{P_{p}}$ and $J(4,4)$ and adding an interval condition which can be justified by mechanical restrictions:

$$
\frac{P_{s}}{3}<\overline{P_{p}}<P_{s}
$$

From this consideration, it can be claimed that :

$$
L<\frac{r T k_{i} S_{m}}{V_{p} m^{2}}\left(\frac{f}{m}+\frac{r T}{V_{p}} k_{c} 10 P_{s} / 3\right)^{2}=L^{\prime}
$$

and:

$$
\begin{gathered}
R>\left(\frac{k}{m} \frac{r T}{V_{p}} k_{c} 4 P_{s}+\right. \\
\left.\frac{r T}{V_{p}} \frac{k_{s}}{m} S_{p} P_{S}\right)^{2}+\left(\frac{k}{m} \frac{r T}{V_{p}} k_{c} 2 P_{s}+\frac{r T}{V_{p}} \frac{k_{s}}{m} S_{p} P_{S}\right) \times \\
\left(\frac{f}{m} \frac{r T}{V_{p}} k_{c} 2 P_{S}+\frac{k}{m}\right) \times\left(\frac{f}{m}+\frac{r T}{V_{p}} k_{c} 2 P_{s}\right)=R^{\prime}
\end{gathered}
$$
then:

A sufficient condition for stability for all $P_{p}$ is

$$
L^{\prime}<R^{\prime}
$$

Clearly, in order to satisfy (32), $k_{i}$ and $S_{m}$, which appear only in $L^{\prime}$, can be modified. It is also possible to show that for the current parameter values, $R^{\prime}$ is increasing with $k$.

From stability conditions (26) and (32), we conclude that among all the possibilities of parameter shift, an increase of $k$ can lead to stability for the system.

\section{EXPERIMENTAL AND SIMULATION RESULTS}

In this section, we compare experimental and simulation results before and after the increase of the spring's stiffness. Increasing $k$ is one mechanical simple operation since it just requires a replacement of the comparator's spring.

The exact value of $P_{p}$ and approximate value of $x$ are available through experiments. This enables us to compare the averaged trajectory of the pressures with the simulation.

Experiments and simulations were performed for a linear negative ramp demand $W_{p}$. They show the multiplication of $k$ by 2 actually works pretty well since after a modification of the spring value, the regulator stopped chattering. This can be clearly seen in Fig. 2.

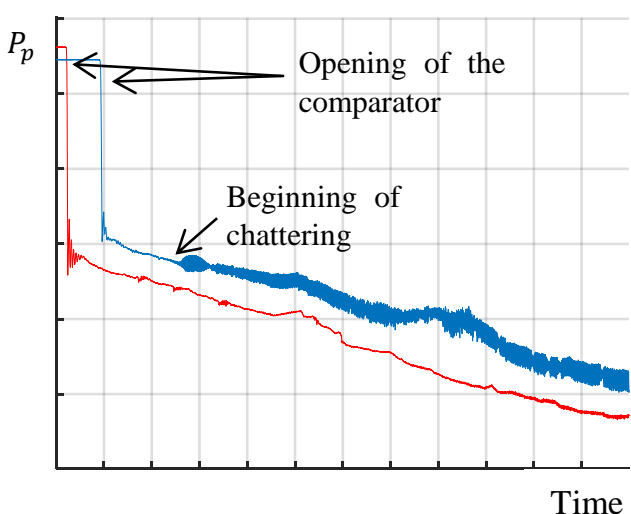

Figure 2: Measurements of $P_{p}$ before (blue) and after (red) the modification of the spring's stiffness.

We precise that no numerical values are given due to confidentiality reasons.

Fig. 3 shows noisy measurements of $P_{m}$ (in red) with a smaller envelope using the modified spring ; the spectrum of $P_{m}$ plotted on Fig. 4 confirms that chattering was eradicated since the three frequency peaks at 32, 64 and $128 \mathrm{~Hz}$ have vanished leaving only noise due to the sensor and the flow recirculation. Note that the spectrum has been plotted here for the same constant mass flow demand $W_{p}$. This comparison remains the same for all mass flows, excepted that the regulator endeavors the well- known phenomenon of period adding before the modification (see Budd, 1996).

Simulation results plotted on Fig. 5 show that chattering of $P_{m}$ is barely visible at the end of the blue curve, while it doesn't exist after the modification, whereas it cannot be seen on the curves of $P_{p}$ on Fig. 6.

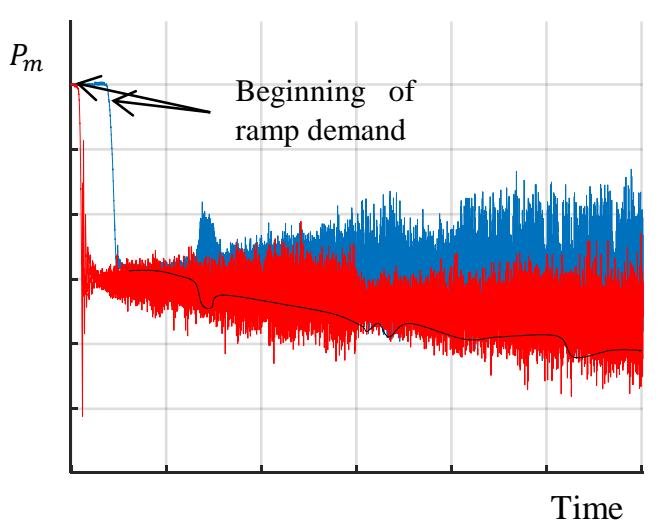

Figure 3: Measurements of $P_{m}$ before (blue) and after (red) the modification of the spring's stiffness. The black line highlights the envelope of the blue curve. 


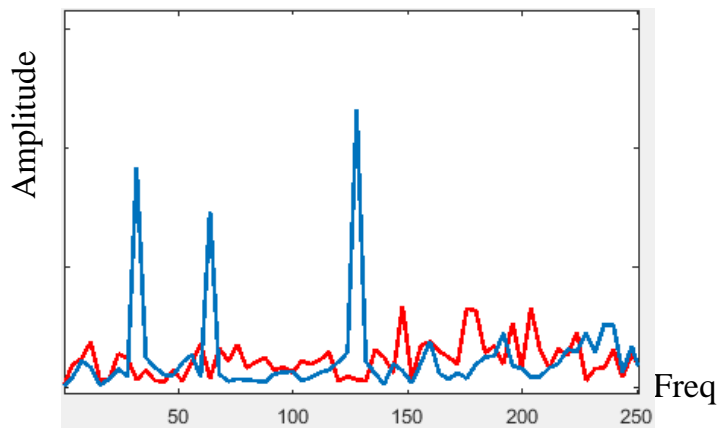

Figure 4: Amplitude spectrum of $P_{m}$ before (blue) and after (red) the modification of the spring's stiffness (x-axis represents the frequency in $\mathrm{Hz}$ ).

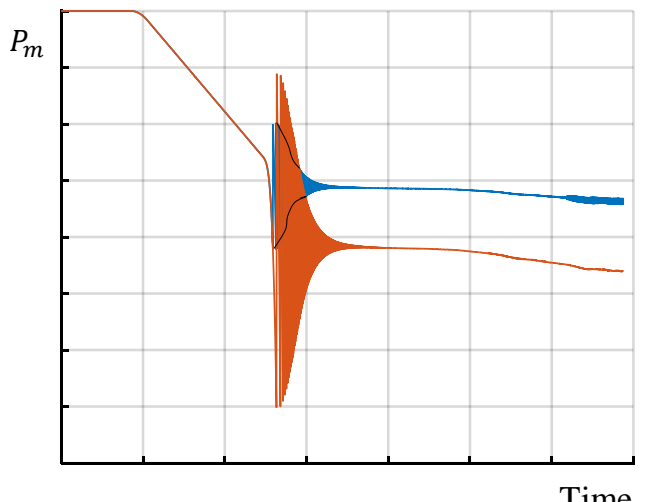

Time

Figure 5: Simulation results for $P_{m}$ before (blue) and after (red) the modification of the spring's stiffness. The black lines highlight the envelope of the blue curve.

In addition a good way to show the chattering is to plot the displacement $x$ of the comparator (Fig. 7), which is touching its support when $x$ goes to zero.

It is also possible to plot the eigenvalues of the linearized model at regularly increasing time samples when the ramp demand $W_{p}$ decreases slowly after the convergence of the transient oscillations and see how they move. Before modification (Fig. 8), there are two positive real part eigenvalues showing the instability of the system. These two eigenvalues remains in the left-half plane after the modification of $k$ (Fig. 9). This fact confirms that the system has been stabilized.

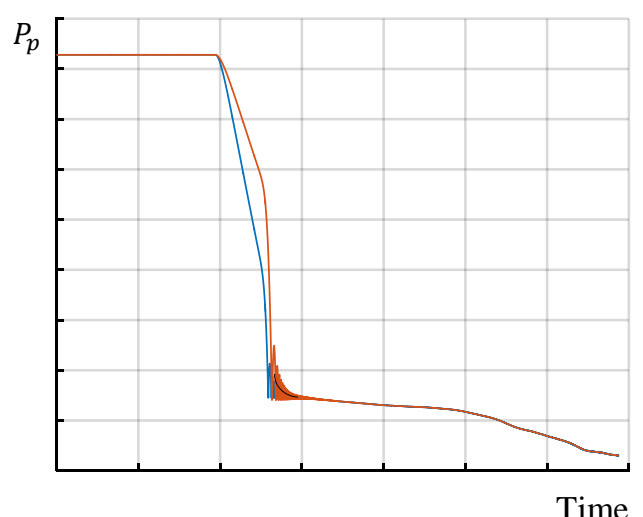

Time

Figure 6: Simulation results for $P_{p}$ before (blue) and after (red) the modification of the spring's stiffness. The black line highlights the envelope of the blue curve.

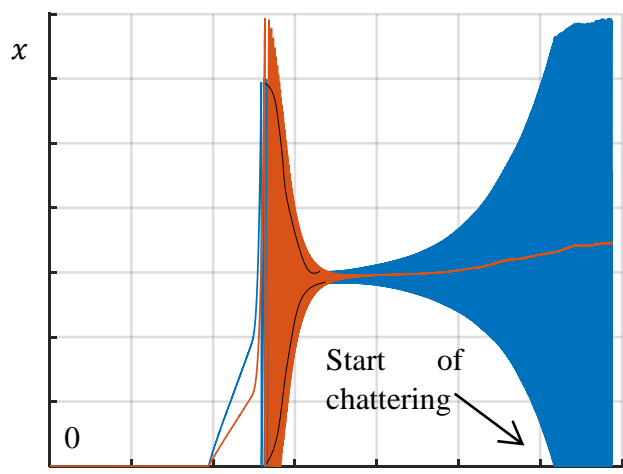

Time

Figure 7: Simulation results for $x$ before (blue) and after (red) the modification of the spring's stiffness. (measurements for $\mathrm{x}$ and $\dot{x}$ are not available). The black lines highlight the envelope of the blue curve.

Another consequence of this manipulation can be observed through the transient oscillations, which can be seen on Fig. 2, 3, 5 and 6 at the end of the early fall of pressures. Their envelope have been increased with $k$. This means that the mask pressure will oscillate a bit more just after the opening of the comparator even if the chattering is suppressed. This may disturb the pilot at the beginning of an inhalation, and a nonlinear analysis could be performed to see to which parameters the amplitude of the opening oscillations are the most sensitive and if the modification of the former to attenuate the latter still respect the stability conditions. 


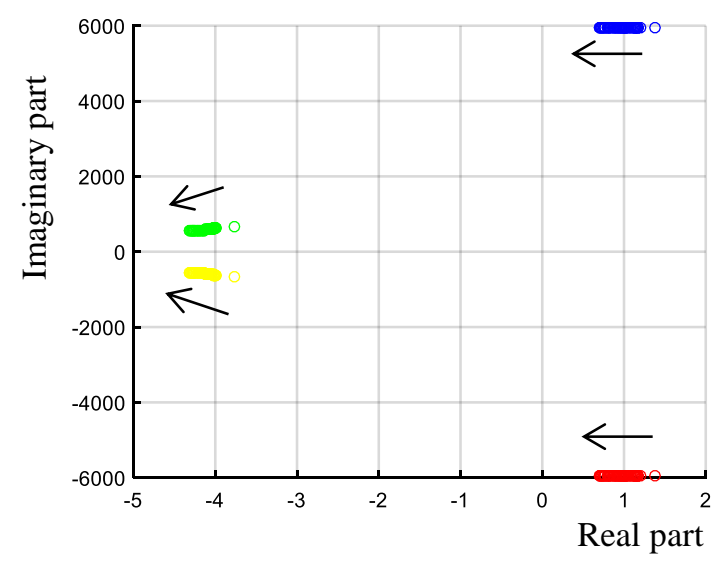

Figure 8: Eigenvalues of the system before modification. The arrows show the sense of evolution with the ramp demand.

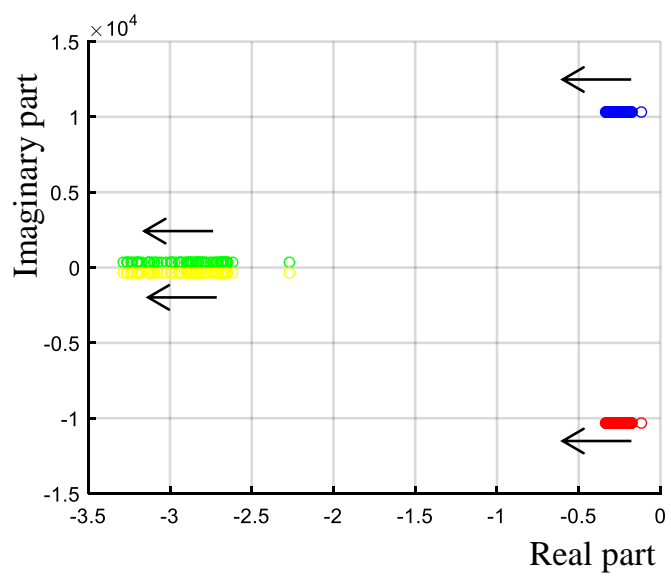

Figure 9: Eigenvalues of the system after modification. The arrows show the direction of evolution with the ramp demand.

\section{MATHEMATICAL ANALYSIS OF THE SIMULATION RESULTS}

It is possible to come back on some observations made in the previous paragraph by looking at the equilibrium equations (8)-(9)-(10). Parameter $k$ does not appear in equations (8) and (9), that is the equilibrium values for $x$ and $P_{p}$ remain the same whatever $k$. This explains why pressures before and after modification superpose each other on Fig. 6.

From (9), $\bar{x}$ increases while $\overline{P_{p}}$ decreases since its derivative is strictly negative:

$$
\frac{d \bar{x}}{d \overline{P_{p}}}=-\frac{k_{c}}{k_{s}}\left(\frac{P_{s}^{2}}{{\overline{P_{p}}}^{2}}+1\right)<0
$$

From (14), with an increase of $k$, the value of $P_{m}$ decreases. This is coherent with Fig. 5.

From numerical values, the determinant of the characteristic equation corresponding to equation (2) is $f^{2}-4 k m<0$. Before and after the modification, the mechanical part of the system presents more or less damped poles. This partly explains the oscillations of $x, P_{p}$ and $P_{m}$ (indeed, equation (2) is not a simple second order linear differential equation since it is perturbed by $P_{p}$ through the nonlinear dynamics of the whole system). The determinant becomes even more negative by increasing $k$, which goes in the way of having more transient oscillations. Note that the transient oscillations are a different from the chattering effect. Since at the equilibrium, $P_{m}$ has been lowered after the modification, it takes more time for $P_{m}$ to reach this new value, which explains the result of Fig. 5. The consequence in practice is a bigger difficulty to breathe in the mask.

Besides the longer fall of $P_{m}$, we can note also a longer fall of $P_{p}$, more difficult to analyse mathematically due to the coupling of equations (4) and (7). A nonlinear analysis would be necessary to understand how the fall of $P_{p}$ works.

All these elements show that after the modification, the regulation will send oxygen later (due to the longer decrease of $P_{p}$ ), the mask will be more difficult to breathe in (decrease of $P_{m}$ ), with more transient oscillations.

\section{CONCLUSION}

After proposing a mathematical model for a regulated oxygen mask, we performed a linear stability analysis around its equilibrium points. Thanks to the Routh criterion we found conditions to prevent the regulated oxygen mask from chattering by assuring its stability, and deduced which parameters can be modified to fulfil it. The easiest action is to increase the stiffness of the comparator and we experimentally showed that performing this action was sufficient to get rid of the chattering effect. However, the counterpart of the suppression of chattering is a bigger difficulty to breathe in the mask and the increase of the envelope of opening oscillations. Further studies of the nonlinear dynamics of this system could help us finding the sensitivity of the opening oscillations to some parameters that could be compared with the 
stability conditions of the Routh criterion. If these conditions were contradictory, a compromise should be found, based on the feeling of the breathing pilot.

\section{REFERENCES}

Bernardo, M., Budd, C., Champneys, A. R., \& Kowalczyk, P. (2008), Piecewise-smooth dynamical systems: theory and applications, Vol. 163, Springer-Verlag, London.

Budd, C. J. (1996), "Non-smooth dynamical systems and the grazing bifurcation", Nonlinear mathematics and its applications, Guildford 1995, Cambridge University Press, Cambridge, 219-235.

Chin, W., Ott, E., Nusse, H. E., \& Grebogi, C. (1994), "Grazing bifurcations in impact oscillators", Physical Review E 50(6), 4427-4444.

Casas, F., Chin, W., Grebogi, C., \& Ott, E. (1996), "Universal grazing bifurcations in impact oscillators", Physical review E 53(1), pp. 134.

EASA (2012). "Certification Specifications for Large Aeroplanes CS-25", available at: https://www.easa.europa.eu/system/files/dfu/CS25_Amdt\%203_19.09.07_Consolidated\%20version.pd $\underline{\mathrm{f}}$

Hayashi, S., Hayase, T., \& Kurahashi, T. (1997), "Chaos in a hydraulic control valve", Journal of fluids and structures, 11(6), 693-716.

Hös C.J., Champneys A.R., et al (2017), "Dynamic behaviour of direct spring loaded pressure relief valves connected to inlet piping: IV review and recommendations", Journal of Loss Prevention in the Process Industries, Volume 48, 2017, 270-288.

Licsko, G., Champneys, A., Hos, C. (2009), "Nonlinear Analysis of a Single Stage Pressure Relief Valve", International journal of applied mathematics, 39(4), $12-26$.

Maeda, T. (1970), "Studies on the dynamic characteristic of a poppet valve: 1 st report, theoretical analysis", Bulletin of JSME, 13(56), 281-289.

McCloy, D. and Martin, H.R. (1980), Control of fluid power: analysis and design, Chichester, Sussex, England, Ellis Horwood, Ltd. New York, Halsted Press.

MacLeod, G. (1985), "Safety valve dynamic instability: an analysis of chatter", J. Pressure Vessel Technol 107(2), 172-177.

Molenaar, J., de Weger, J. G., \& van de Water, W. (2001), "Mappings of grazing-impact oscillators", Nonlinearity, 14(2), 301.

NATO (1991). "Functional Requirements of aircraft oxygen equipment and pressure suits STANAG 3198", available http://standards.globalspec.com/std/399286/nato$\underline{\text { stanag-3198. }}$ 